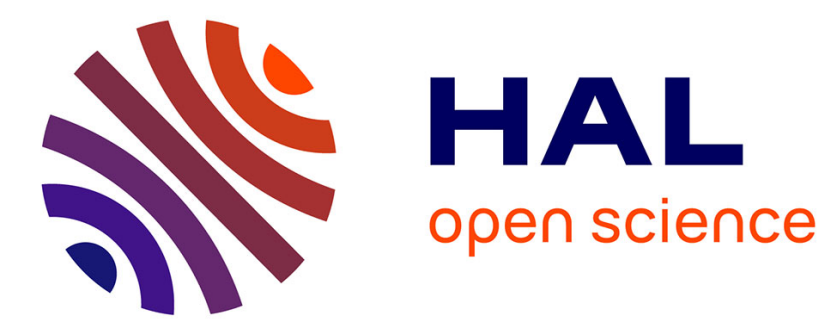

\title{
Lymph node management in clinically node-negative patients with papillary thyroid carcinoma
}

\author{
S. Vergez, J. Sarini, J. Percodani, E. Serrano, Ph Caron
}

\section{To cite this version:}

S. Vergez, J. Sarini, J. Percodani, E. Serrano, Ph Caron. Lymph node management in clinically nodenegative patients with papillary thyroid carcinoma. EJSO - European Journal of Surgical Oncology, 2010, 36 (8), pp.777. 10.1016/j.ejso.2010.06.015 . hal-00608923

\section{HAL Id: hal-00608923 \\ https://hal.science/hal-00608923}

Submitted on 16 Jul 2011

HAL is a multi-disciplinary open access archive for the deposit and dissemination of scientific research documents, whether they are published or not. The documents may come from teaching and research institutions in France or abroad, or from public or private research centers.
L'archive ouverte pluridisciplinaire HAL, est destinée au dépôt et à la diffusion de documents scientifiques de niveau recherche, publiés ou non, émanant des établissements d'enseignement et de recherche français ou étrangers, des laboratoires publics ou privés. 


\section{Accepted Manuscript}

Title: Lymph node management in clinically node-negative patients with papillary thyroid carcinoma

Authors: S. Vergez, J. Sarini, J. Percodani, E. Serrano, Ph Caron

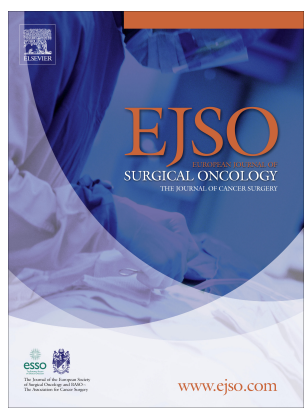

PII:

S0748-7983(10)00191-5

DOI:

10.1016/j.ejso.2010.06.015

Reference: YEJSO 3003

To appear in: European Journal of Surgical Oncology

Received Date: 4 May 2009

Revised Date: 24 December 2009

Accepted Date: 7 June 2010

Please cite this article as: Vergez S, Sarini J, Percodani J, Serrano E, Caron P. Lymph node management in clinically node-negative patients with papillary thyroid carcinoma, European Journal of Surgical Oncology (2010), doi: 10.1016/j.ejso.2010.06.015

This is a PDF file of an unedited manuscript that has been accepted for publication. As a service to our customers we are providing this early version of the manuscript. The manuscript will undergo copyediting, typesetting, and review of the resulting proof before it is published in its final form. Please note that during the production process errors may be discovered which could affect the content, and all legal disclaimers that apply to the journal pertain. 
Original article

\section{Lymph node management in clinically node-negative patients with papillary thyroid carcinoma}

S Vergez ${ }^{1}$, J Sarini ${ }^{2}$, J Percodani ${ }^{1}$, E Serrano ${ }^{1}$, Ph Caron ${ }^{3}$

${ }^{1}$ Department of Head and Neck Surgery, University Hospital Rangueil-Larrey, Toulouse, France

${ }^{2}$ Department of Surgical Oncology, Institut Claudius Regaud, Toulouse, France

${ }^{3 .}$ Department of Endocrinology, University Hospital Rangueil-Larrey, Toulouse, France

Reprint requests: Dr Sebastien Vergez,

Service ORL, Centre Hospitalo-Universitaire Rangueil-Larrey,

24 chemin de Pouvourville

31059 Toulouse cedex 9

France

Phone. +33567771789

vergez.s@chu-toulouse.fr

A part of the results was presented as an oral communication at the $32^{\text {nd }}$ annual meeting of the European Thyroid Association, Leipzig, September $4^{\text {th }}, 2007$. 


\section{Abstract}

Aims: Systematic lymph node dissection in patients with papillary thyroid carcinoma (PTC) remains controversial. The objective of this study was to study the pattern of lymph node spread in patients with PTC clinically node-negative and then to propose a lymph node management strategy.

Methods: We retrospectively reviewed the records of patients who had undergone total thyroidectomy and a systematic central neck dissection (CND) and lateral neck dissection. Ninety patients with PTC without lymph nodes metastases (LNM) detected on preoperative palpation and ultrasonographic examination were included.

Results: Forty-one patients (45.5\%) had LNM. Twenty-eight patients (31\%) had a central and a lateral involvement. Thirteen patients (14.5\%) had only a central involvement. All the patients without LNM in the central compartment were also free in the lateral compartment. There was no correlation between LNM status and TNM staging.

The largest LNM in the central compartment was smaller than or equal to $5 \mathrm{~mm}$ in $66 \%$ of the cases, and that could explain the lack of sensitivity of the preoperative ultrasonographic examination.

Conclusion: CND could be considered at preoperative or intraoperative diagnosis of PTC whereas lateral neck dissection should be performed only in patients with preoperative suspected and/or intraoperatively proven LNM. Systematic CND allows an objective evaluation of lymph node status in this central cervical area where the LNM are particularly small and difficult to detect preoperatively.

Keywords: papillary thyroid carcinoma, lymph node metastases, central neck dissection, selected neck dissection.

\section{Introduction}

Systematic lymph node dissection in patients with papillary thyroid carcinoma (PTC) is controversial. Cervical areas are the first sites of the recurrence of PTC [1]. The impact of lymph node metastasis (LNM) on the prognosis of papillary disease regarding survival without recurrence and overall survival determines the extent of surgery. Without preoperatively diagnosed LNM, the indications and the appropriate extents of central neck dissection (CND, compartment VI) and/or lateral neck dissections with their respective morbidity are debated and attitudes vary according to authors.

In patients with differentiated thyroid carcinoma, Hughes et al in a matched-pair analysis $(n=200)$, and Lin et al, in a retrospective study $(\mathrm{n}=1013)$, have reported a correlation between node invasion and an increased rate 
of local recurrence $[2,3]$. However, in these studies LNM did not influence the survival rate [2, 3]. Lundgren et al, in a nested case-control study of 5123 patients with differentiated thyroid carcinoma, reported a higher mortality in the pN1 group [4]. Therefore the impact of neck node dissection on the prognosis is controversial. For some authors, the prognosis is not influenced by node surgery [5] whereas for others, neck node dissection significantly improves overall survival $[6,7]$.

The heterogeneity of these results assessing the prognostic impact of the LNM as well as dissection, combined with a lack of prospective analysis, explains the lack of consensus concerning the nodal management of PTC. In the case of cervical LNM, either suspected or proven by fine needle aspiration, it is commonly accepted that the surgery may not be limited to a Berry-Picking procedure [1, 6, 8, 9]. A dissection is systematically performed, but the limits differ from one centre to another. Noguchi and Murakami, as well as Uchino et al., performed a modified radical neck dissection (MRND) at the central and lateral levels (II, III, IV, and V), respecting the internal jugular vein, the spinal accessory nerve and the sternomastoid $[1,8]$. For the past forty years, Palazzo et al. has described a move away from the Picking and MRND towards a selective lateral neck dissection (SLND) adapted to the invasion, and most of the time concerning levels VI, III and IV [9]. Caron et al. also opted for selective dissections, systematically taking levels III and IV, and extended to other areas according to preoperative clinical and radiological assessment [10].

Our work was focused on the lymph node management in patients with PTC, exclusively in the absence of LNM diagnosed at the initial clinical and ultrasonographic (US) assessment. In our medical teams, before the European consensus [11], we chose to routinely do bilateral CND and ipsilateral lateral neck dissection if there was no suspicious lymph nodes (cN0 patients). A bilateral lateral neck dissection was done in the presence of multiple, bilateral or isthmic tumors. The objective of our work was to evaluate the pattern of lymph node invasions in the central and lateral cervical compartments in patients with PTC and then to propose a lymph node management strategy.

\section{Patients and methods}

\section{Selection of patients.}

This retrospective study was carried out in Toulouse, France, in the Head and Neck surgery department of the Rangueil-Larrey University Hospital and the Claudius Regaud Institute. From 2000 to 2006, and before the European consensus [11], the files from all patients who had a neck node dissection for a PTC were compiled. 
From this exhaustive collection of 230 patients, those who had had a central and a lateral cervical dissection, without any preoperative diagnosis of LNM, were collected, resulting in 90 patients being included.

The selected criteria of inclusion gathered the histopathological diagnosis of the PTC (greater than $10 \mathrm{~mm}$ in size), whatever the stage and differentiation, with a clinically negative neck (cN0). There were no clinical or US signs suggesting LNM in the preoperative assessment. A precessive LNM (cN1) or a metastasis was considered as exclusion criteria in our study. Moreover, when the time between definitive thyroid histological diagnosis and complementary node surgery exceeded six months, we excluded the subjects.

Some patients underwent a total thyroidectomy with a bilateral central and a uni- or bilateral lateral neck dissection at the same time in the case of a preoperative or intraoperative diagnosis of PTC $(n=28)$. A bilateral lateral neck dissection was done in the presence of multiple, bilateral or isthmic tumours. For the other patients $(n=62)$, the diagnosis of PTC was done secondarily and the average time between the lobectomy or thyroidectomy and the cervical dissection was 42 days.

All the surgical reports were analyzed, alongside all the pathology reports. The TNM stage was set for each patient, according to the AJCC 2002 classification. The number, the size and the localization of the tumours were detailed, and compared with the presence of cervical LNM. The number of LNM, with or without extracapsular spread, as well as their size was related to the number of lymph nodes dissected in each cervical area. The complications were also noted and hypoparathyroidism was considered definitive when requiring vitamin D supplementation for more than 6 months. Permanent laryngeal nerve palsy was considered definitive when persisting for more than 6 months.

\section{Surgical procedures.}

All the patients routinely underwent bilateral CND (compartment VI). After having identified and preserved the recurrent laryngeal nerve and the parathyroid glands, the node clearance was performed laterally to the carotid sheaths, caudally to the innominate artery, dorsally to the prevertebral fascia and cranially to the hyoid bone. Thus, the paratracheal, pretracheal, prelaryngeal and superior mediastinal node sites were dissected. In cases of reoperation of the central compartment, the recurrent nerve was found below the sclerosis of the previous surgery (near the innominate artery on the right side and adjacent to the trachea and the oesophagus on the left side).

The selective lateral neck dissection (SLND) systematically preserved the internal jugular vein, the sternomastoid muscle and the spinal accessory nerve. It systematically covered levels II, III and IV respectively, 
the upper, mid and lower jugular groups. Forty-five of the 90 patients operated on by one of the surgical teams also had a level $\mathrm{V}$ node dissection. We accessed these areas by two routes: a J incision along the medial edge of the sternomastoid, or a prolonged Kocher incision, that could be supplemented if necessary by a second high cervical parallel incision (Mac Fee modified). The statistical analysis included an ANOVA test with the StatView $($ software version 4.55. Differences were considered significant when $\mathrm{p}<0.05$.

\section{Results}

\section{Clinical characteristics}

The patient population consisted of 71 women and 19 men, with a mean age of $45+/-16$ [range $13-89$ ] (Table1). According to the TNM classification AJCC 2002 our population was composed of 30 pT1, 30 pT2 and 29 pT3. There was no tumour classified pT4 in our cohort. However, the detailed pathological description was missing for one patient (Table 1).

The CND was bilateral in 86 patients. In 4 patients, the surgical exploration could not be bilateral because of a major local sclerosis. An average of $4.7+/-0.4$ [range 0-21] lymph nodes on the right side and $4.4+/-0.37$ [range 0-22] on the left side had been removed. The proportion of right or left dissections without lymph nodes did not exceed 3 patients and systematically occurred during a second surgical intervention.

The SLND was composed of about 25 lymph nodes +/- 1.1 [range 6-54]. Nineteen patients had bilateral SLND due to the median nature of a single location or the bilateral nature of multiple locations. Sixty-three right dissections and 46 left dissections were done.

\section{Pathological characteristics}

Forty-one patients (i.e. 45.5\%) had lymph node invasion (pN1) whereas neck palpation and ultrasound examination were negative at the assessment preceding the thyroid surgery. Among them, 28 (i.e. $31 \%$ ) had metastatic invasion of the central compartment and the lateral compartment. For 13 patients (i.e. 14.5\%), only the central compartment was the location of LNM. No patient presented a lateral lymph node invasion without a central invasion. Statistical analyses revealed that the patient's age, gender, tumour size and stage of TNM did not influence the risk of node invasion.

Among 28 patients pN1 in the lateral compartment, LNM were found in level II, III, and IV respectively for 15, 16 and 16 patients $(54 \%, 57 \%$ and $57 \%$ of cases). For five patients, the lateral compartment invasion was limited 
to the upper jugular compartment (Level II). Among patients with at least one metastasis in the lateral compartment, 18 patients had an exploration at the posterior triangular level (V) and 9 of them had LNM in this area. A bilateral invasion of the central compartment was found in 18 patients (44\% of pN1 patients). Six patients had bilateral invasion of the lateral compartments. Four patients had contralateral LNM in regard to the thyroid tumour. For one of them, a bilateral multifocal tumour with clear left predominance led to a left central and right lateral invasion. Unilateral tumours of three other patients were associated with contralateral central metastases and ipsilateral cervicolateral ones.

A total of $213 \mathrm{LNM}$ were identified in the central compartment [average $5.2+/-0.8$ per patient]. The average size of central LNM was $4.7 \mathrm{~mm}+/-0.9$ [range $0.5-30 \mathrm{~mm}$ ]. Eighty one percent of invaded LNM were smaller than or equal to $5 \mathrm{~mm}, 16 \%$ were between 5 and $10 \mathrm{~mm}$ and $3 \%$ greater than $10 \mathrm{~mm}$. We concentrated on the comparison of the maximum size of the removed LNM for each patient. While the exact size of each LNM was not always mentioned, the minimum and maximum sizes of the invaded lymph nodes were regularly recorded. The proportion of LNM (in the central compartment) less than or equal to $5 \mathrm{~mm}$ was $66 \%$ while those strictly above $10 \mathrm{~mm}$ remained low at 5\% (Table 2). The average size of LNM with extracapsular spread was $4.9 \mathrm{~mm}$ +/- 1.9 [range 1-15 mm].

A total of 136 LNM were counted with an average number of lateral neck dissections of $4.9+/-0.9$. The average size of invaded lateral LNM was $7.9 \mathrm{~mm}+/-2.1$ [range $0.5-40 \mathrm{~mm}$ ]. The analysis of the maximal size of invaded lateral lymph nodes gave $7 \%$ of LNM inferior or equal to $5 \mathrm{~mm}$, $93 \%$ of LNM superior to $5 \mathrm{~mm}$ (Table 2).

\section{Complications}

One patient presented a definitive laryngeal nerve palsy (i.e. 1\%). This patient needed a reoperation for a contralateral lobectomy with lymph node dissections and was pNO. Six patients had a definitive hypoparathyroidism (i.e. $6.7 \%$ ). Three of them had had a single operation and three others had been reoperated. There was no significant difference between the morbidity of $\mathrm{pN} 0$ and $\mathrm{pN} 1$ patients (Table 1).

\section{Follow-up}

The median follow-up was 62 months [14-105]. Ten patients who presented LNM (or a tumor, n=1) with a large extracapsular spread had had an external radiation therapy. Each patient received a radio-iodine treatment (90/90). This treatment needed to be repeated for six patients (initially pN1 with a detectable thyroglobulin blood levels during the follow-up without any radiological target for five of them). They are actually disease-free. 
Three patients died (death unrelated to the disease). Seven patients were lost after the first year of follow-up. Two patients with LNM in central compartment had had another dissection for a node recurrence and are disease-free at the last evaluation. Another patient who was initially pN0 developed recently a small and unique lateral LNM eight years after the treatment. The seventy-one other patients are disease-free since the first treatment.

\section{Discussion}

\section{LNM staging}

Preoperative node invasion is often underestimated in papillary disease. In our study, $41 / 90 \mathrm{cN} 0$ patients actually had LNM. According to data from the literature, 38 to $90 \%$ of nodal metastases are not clinically diagnosed [12]. The proportion of non-diagnosed LNM in our study could have been explained by the size of nodal metastases, often less than $5 \mathrm{~mm}$. On the contrary, few non-diagnosed LNM were above $10 \mathrm{~mm}$ but the US examination was done before the initial consultation and some patients had the node dissection a few weeks after the definitive pathological diagnosis of PTC. In this context, careful cervical palpation and a detailed cervical ultrasound examination are carried out preoperatively to search for central, jugular and spinal lymph nodes. A fine needle aspiration of lymphadenopathy for cytology can be done, possibly ultrasound-guided, combined with a measure of thyroglobulin concentration. Cystic appearance, hyperechoic punctuations, loss of hilum, and peripheral vascularization can be considered as major US criteria of LNM [13]. It is interesting to note that in our results, central LNM were clearly smaller than lateral LNM, with most of the central LNM measuring less than $5 \mathrm{~mm}$. The sensitivity of the US diagnosis of central LNM described in literature varies from $10.5 \%$ to $29 \%$ [14, 15$]$. Sugitani et al. found a positive predictive value of $98 \%$ for the lateral compartment $(n=130)$ [15]. Therefore a preoperative clinical or ultrasonographic diagnosis of LNM is particularly difficult in the central compartment, reinforcing the rationale of a systematic CND.

\section{Central compartment LNM}

In our patients, central node invasion was present in $45.5 \%$ of the patients. The central compartment is the first lymph node site in thyroid cancer [1,9], except in rare cases of superior polar tumours in which a jugular chain drainage is first described [16]. This drainage frequently crosses at the central compartment [16]. In our study, a bilateral central nodal invasion was observed in 18 patients (i.e. $44 \%$ of pN1 patients), and 3 patients had a unilateral central invasion, contralateral to a unique tumour. Central invasion is frequent, precocious and non- 
lateralized, leading to the suggestion that a bilateral CND should be combined with a total thyroidectomy after the diagnosis of PTC [17-19]. In a retrospective analysis, with an average follow-up of $13.3+/-6.2$ years $(\mathrm{n}=$ 195), Tisell et al. highlighted an improvement of survival, brought about by a prophylactic central dissection [6]. However, the CND procedure increases the morbidity observed after a thyroidectomy alone [20, 21]. Permanent laryngeal nerve palsy occurs with a higher incidence: 0 to $4.8 \%$ [18, 21-22]. CND significantly reduces serum parathyroid hormone levels [22]. Our hypoparathyroidism rates are in the upper range of the published rates. Definitive hypoparathyroidism after CND has been described as being from $0.9 \%$ to $11.3 \%$ [14, 18, 21-23]. In our study there was no significant difference seen when this dissection was done at a later date, but many authors report that this increases complications related to this surgery [21-24]. And our unique definitive laryngeal nerve palsy was observed after a reoperation.

The European consensus acknowledges that a prophylactic CND allows an accurate staging of the disease and may guide subsequent treatment and follow-up, but it also specifies that its benefits remain controversial [11]. On the other hand, the American Thyroid Association recent guidelines argue that a prophylactic CND should be considered for patients with PTC especially for advanced primary tumors (T3 or T4) [25]. Evidence-based recommendations support CND for PTC in patients under the care of experienced endocrine surgeons [20]. Therefore we opted for a systematic bilateral CND, allowing an objective LNM staging.

\section{Lateral compartment LNM}

Bonnet et al. purposed a prophylactic neck dissection including central and ipsilateral III and IV (eventually extended to II and V) for PTC less than $2 \mathrm{~cm}$. This LNM staging modified the indication for radioiodine ablation for $30 \%$ of patients [23]. Pereira et al. reported the lack of central recurrence in a group of patients who underwent a prophylactic central dissection, and described a correlation between an involvement in the lateral compartment (group of patients without prophylactic lateral dissection) and an initial central invasion including more than five LNM. No lateral disease in their series occurred in pN0 or pN1 patients who had less than five LNM [26]. Goropoulos et al. decided to extend the node dissection into the lateral neck compartments depending on the histological results of the central compartment. Without a preoperative diagnosis of central invasion, the decision is made on the basis of the results of the analysis of frozen sections from CND [27].

We found no lateral nodal involvement without a central involvement, but in the literature, the presence of lateral LNM without combined central invasion (skip metastases) has been regularly reported: Noguchi and Murakami 7\%, Chung et al. 7.7\%, Bonnet et al. 12.5\%, Ito et al. 12.9\% and Mirallie et al. $15.3 \%$ [1, 12, 14, 23, 28]. 
A study conducted in a cohort of 2966 patients helped to highlight the gain in terms of survival provided by an MRND in the context of extrathyroid tumour invasion or large node invasions [7]. Ohshima et al. even suggested that a systematic prophylactic bilateral MRND should be carried out in the presence of risk factors, such as bulky, bilateral or isthmic tumours. In their series they decreased the proportion of distant metastases, the number of cancer deaths and improved the rate of survival at 10 years [29]. However, in the absence of LNM diagnosed during initial assessment, many authors do not perform any prophylactic node surgery [9, 10]. In our study 62/90 patients had had an SLND without involvement in the lateral compartment and therefore we have now decided not to perform systematic SLND because the lateral compartment can easily be followed by ultrasound [15] and a SLND can be performed subsequently without the increased morbidity if an LNM (skipmetastasis or not) appears. Based on these results, we do not then expose every patient with PTC to the morbidity and the scar of a systematic SLND. But, in the cases of large extrathyroid invasion or large LNM in the central compartment, we perform an ipsilateral SLND.

In Sivanandan and Soo's studies, the proportion of invaded node levels in $\mathrm{cN} 1$ patients, confirmed the preferential invasion of middle and low jugular areas (levels III and IV, in 50 to 72\%) [30]. Nevertheless, they also recorded a non-negligible proportion of posterior triangular nodes (level $\mathrm{V}$ in 24 to $44 \%$ ), and upper jugular (level II in 45 to $56 \%$ ) involvement. In our study, this upper jugular and posterior triangular invasion were found in $54 \%$ and $50 \%$ respectively of $\mathrm{pN} 1$ patients laterally. Therefore, when a therapeutic SLND is indicated, we systematically pick out levels II to $\mathrm{V}$.

\section{Conclusion}

This clinical study suggests that systematic bilateral central node dissection is indicated in patients with PTC (more than $1 \mathrm{~cm}$ in size). In case of preoperative or intraoperative diagnosis of PTC, this CND is performed at the time of the total thyroidectomy and enables an objective evaluation of the lymph node status to be made. The small diameter of the LNM of the central compartment explains the lack of sensitivity of the preoperative ultrasonographic assessment.

On the other hand, SLND is unnecessary without a clinical and/or US diagnosis of lymph node involvement at the initial assessment. This suggestion is based upon an initial cervical US which precisely evaluates the lateral cervical compartments (II-V). 
These results reinforce the European consensus, but further prospective studies are necessary in order to analyse the impact on the quality of life and survival without recurrence in patients with PTC treated by total thyroidectomy and CND.

Acknowledgements. The authors thank Dr Delphine Berchery for the statistical analyses, Dr Adil Benlyazid and John Woodley for the English corrections.

Declaration of interest. The authors declare that there is no conflict of interest that could be perceived as prejudicing the impartiality of the research reported.

Funding. This research did not receive any specific grant from any funding agency in the public, commercial or not-for-profit sector. 


\section{References}

1. Noguchi S, Murakami N. The value of lymph-node dissection in patients with differentiated thyroid cancer. Surg Clin North Am 1987; 67:251-61.

2. Hughes CJ, Shaha AR, Shah JP, Loree TR. Impact of lymph node metastasis in differentiated carcinoma of the thyroid: a matched-pair analysis. Head Neck 1996; 18:127-32.

3. Lin JD, Liou MJ, Chao TC, Weng HF, Ho YS. Prognostic variables of papillary and follicular thyroid carcinoma patients with lymph node metastases and without distant metastases. Endocr Relat Cancer 1999; 6:109-15.

4. Lundgren CI, Hall P, Dickman PW, Zedenius J. Clinically significant prognostic factors for differentiated thyroid carcinoma: a population-based, nested case-control study. Cancer 2006; 106:524-31.

5. Steinmuller T, Klupp J, Rayes N et al. Prognostic factors in patients with differentiated thyroid carcinoma. Eur J Surg 2000; 166:29-33.

6. Tisell LE, Nilsson B, Molne J et al. Improved survival of patients with papillary thyroid cancer after surgical microdissection. World J Surg 1996; 20:854-9.

7. Noguchi S, Murakami N, Yamashita H, Toda M, Kawamoto H. Papillary thyroid carcinoma: modified radical neck dissection improves prognosis. Arch Surg 1998; 133:276-80.

8. Uchino S, Noguchi S, Yamashita H, Watanabe S. Modified radical neck dissection for differentiated thyroid cancer: operative technique. World J Surg 2004; 28:1199-203.

9. Palazzo FF, Gosnell J, Savio R et al. Lymphadenectomy for papillary thyroid cancer: changes in practice over four decades. Eur J Surg Oncol 2006; 32:340-4.

10. Caron NR, Tan YY, Ogilvie JB et al. Selective modified radical neck dissection for papillary thyroid canceris level I, II and V dissection always necessary? World J Surg 2006; 30:833-40.

11. Pacini F, Schlumberger M, Dralle $\mathrm{H}$ et al. European consensus for the management of patients with differentiated thyroid carcinoma of the follicular epithelium. Eur J Endocrinol 2006; 154:787-803.

12. Mirallie E, Visset J, Sagan C, Hamy A, Le Bodic MF, Paineau J. Localization of cervical node metastasis of papillary thyroid carcinoma. World J Surg 1999; 23:970-3.

13. Leboulleux S, Girard E, Rose M, et al. Ultrasound criteria of malignancy for cervical lymph nodes in patients followed up for differentiated thyroid cancer. J Clin Endocrinol Metab 2007; 92:3590-4.

14. Ito $\mathrm{Y}$, Tomoda $\mathrm{C}$, Uruno $\mathrm{T}$ et al. Clinical significance of metastasis to the central compartment from papillary microcarcinoma of the thyroid. World J Surg 2006; 30:91-9. 
15. Sugitani I, Fujimoto Y, Yamada K, Yamamoto N. Prospective outcomes of selective lymph node dissection for papillary thyroid carcinoma based on preoperative ultrasonography. World J Surg 2008; 32:2494-502.

16. Attie JN. Modified neck dissection in treatment of thyroid cancer: a safe procedure. Eur J Cancer Clin Oncol $1988 ; 24: 315-24$.

17. Gimm O, Rath FW, Dralle H. Pattern of lymph node metastases in papillary thyroid carcinoma. Br J Surg $1998 ; 85: 252-4$.

18. Koo BS, Choi EC, Yoon YH et al. Predictive factors for ipsilateral or contralateral central lymph node metastasis in unilateral papillary thyroid carcinoma. Ann Surg. 2009; 249:840-4.

19. Lee SH, Lee SS, Jin SM et al. Predictive factors for central compartment lymph node metastasis in thyroid papillary microcarcinoma. Laryngoscope 2008; 118:659-62.

20. White ML, Gauger PG, Doherty GM. Central Lymph Node Dissection in Differentiated Thyroid Cancer. World J Surg 2007; 31:895-904.

21. Filho JG, Kowalski LP. Surgical complications after thyroid surgery performed in a cancer hospital. Otolaryngol Head Neck Surg 2005; 132:490-4.

22. Roh JL, Park JY, Park CI. Total Thyroidectomy Plus Neck Dissection in Differentiated Papillary Thyroid Carcinoma Patients: Pattern of Nodal Metastasis, Morbidity, Recurrence, and Postoperative Levels of Serum Parathyroid Hormone. Ann Surg 2007; 245(4):604-610.

23. Bonnet S, Hartl D, Leboulleux S et al. Prophylactic lymph node dissection for papillary thyroid cancer less than $2 \mathrm{~cm}$ : implications for radioiodine treatment. J Clin Endocrinol Metab. 2009; 94:1162-7.

24. Ito Y, Miyauchi A. Lateral and mediastinal lymph node dissection in differentiated thyroid carcinoma: dications, benefits and risks. World J Surg 2007; 31:905-15.

25. Cooper DS, Doherty GM, Haugen BR et al. Revised american thyroid association management guidelines for patients with thyroid nodules and differentiated thyroid cancer. Thyroid 2009; 19:1167-2142.

26. Pereira JA, Jimeno J, Miquel J et al. Nodal yield, morbidity, and recurrence after central neck dissection for papillary thyroid carcinoma. Surgery 2005; 138:1095-100.

27. Goropoulos A, Karamoshos K, Christodoulou A et al. Value of the cervical compartments in the surgical treatment of papillary thyroid carcinoma. World J Surg 2004; 28:1275-81.

28. Chung YS, Kim JY, Bae JS et al. Lateral lymph node metastasis in papillary thyroid carcinoma: results of a therapeutic lymph node disssection. Thyroid 2009; 19:241-6. 


\section{ACCEPTED MANUSCRIPT}

29. Ohshima A, Yamashita $\mathrm{H}$, Noguchi $\mathrm{S}$ et al. Is a bilateral modified radical neck dissection beneficial for patients with papillary thyroid cancer? Surg Today 2002; 32:1027-30.

30. Sivanandan R, Soo KC. Pattern of cervical lymph node metastases from papillary carcinoma of the thyroid. Br J Surg 2001; 88:1241-4. 
Table 1. Pathological data of the patient population.

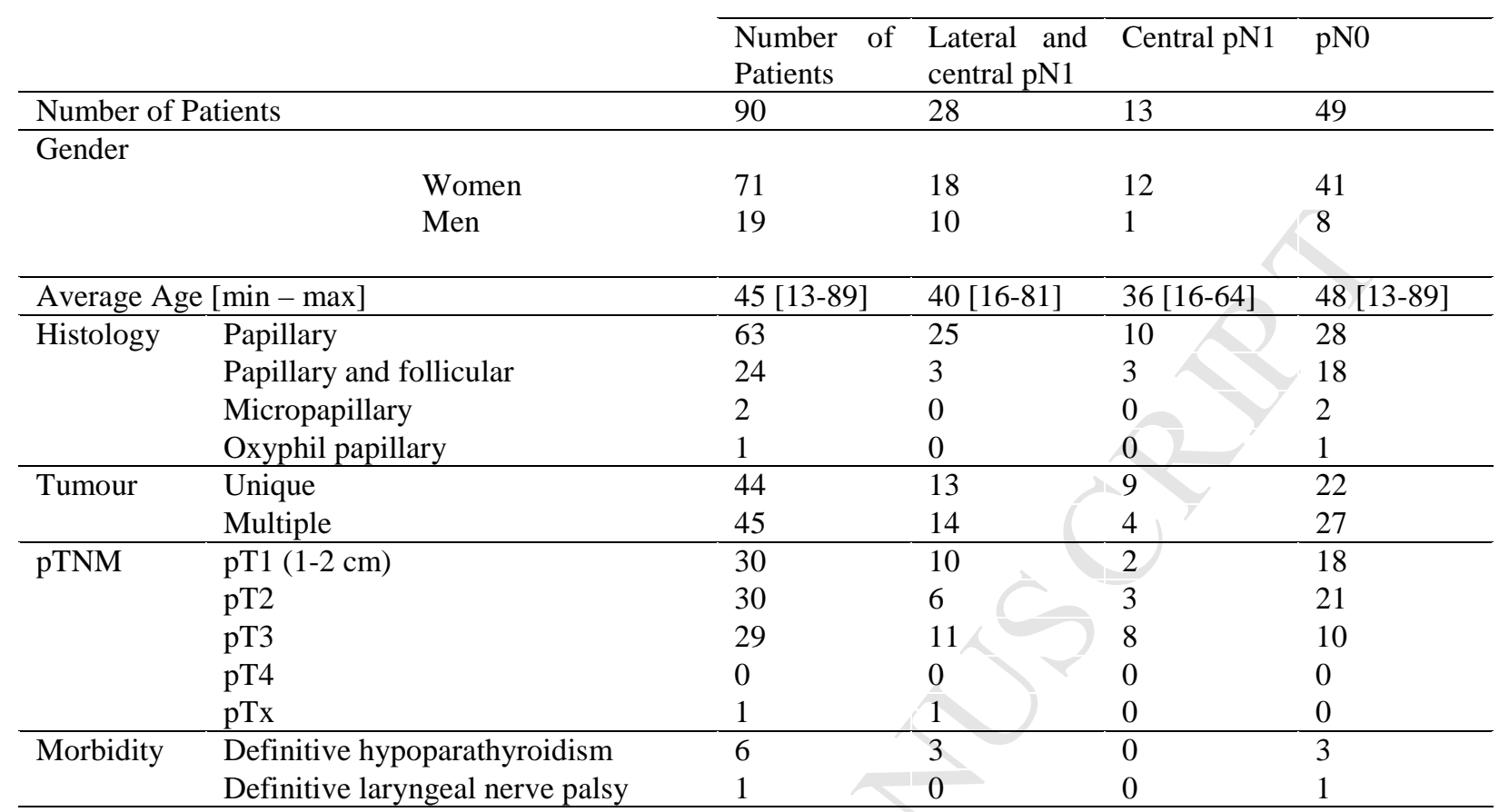


Table 2. Diameters of the largest LNM by compartment.

\begin{tabular}{ll}
\hline $\mathrm{D} \leq 5 \mathrm{~mm}$ & $5 \mathrm{~mm}<\mathrm{D}$ \\
& $\mathrm{D} \leq 10 \mathrm{~mm}$
\end{tabular}

\begin{tabular}{lccc}
\hline Central compartment nodal metastases & $27 / 41(66 \%)$ & $12 / 41(29 \%)$ & $2 / 41(5 \%)$ \\
\hline Lateral compartment nodal metastases & $2 / 28(7 \%)$ & $14 / 28(50 \%)$ & $12 / 28(43 \%)$ \\
\hline
\end{tabular}

